\title{
Lagrange formulation of the symmetric teleparallel gravity
}

\author{
M. Adak \\ Department of Physics, Faculty of Arts and Sciences, \\ Pamukkale University, 20100 Denizli, Turkey \\ madak@pamukkale.edu.tr \\ M. Kalay \\ Department of Physics, Faculty of Arts and Sciences, \\ Pamukkale University, 20100 Denizli, Turkey \\ mkalay@pamukkale.edu.tr \\ Ö. Sert \\ Department of Physics, Faculty of Arts and Sciences, \\ Pamukkale University, 20100 Denizli, Turkey \\ osert@pamukkale.edu.tr
}

\begin{abstract}
We develop a symmetric teleparallel gravity model in a space-time with only the nonmetricity is nonzero, in terms of a Lagrangian quadratic in the non-metricity tensor. We present a detailed discussion of the variations that may be used for any gravitational formulation. We seek Schwarzschild-type solutions because of its observational significance and obtain a class of solutions that includes Schwarzschild-type, Schwarzschild-de Sitter-type and ReissnerNordström-type solutions for certain values of the parameters. We also discuss the physical relevance of these solutions.
\end{abstract}


Although Einstein's (pseudo-)Riemannian formulation of gravitation, the so-called general relativity (GR), is elegant and successful, there are other hints to study non-Riemannian generalizations [1]. In GR, only the dynamical field is the metric or the co-frame and the corresponding field strength is the curvature $R_{b}^{a}$ written in terms of the Levi-Civita connection. In non-Riemannian gravity models the geometry is enriched by adding torsion $T^{a}$ and non-metricity $Q_{b}^{a}$ that modify the Levi-Civita connection $\omega_{b}^{a}$ of the Riemannian space-time (see [1-4] and references therein). The orthonormal components of the metric $\eta_{a b}$, orthonormal co-frame 1 -forms $e^{a}$ and the full connection 1 -fotms $\Lambda_{b}^{a}$ are considered as gauge potentials in the gauge approach to gravity. The corresponding field strengths are defined by the covariant exterior derivatives of the potentials; namely the non-metricity, torsion and curvature, respectively. The most general non-Riemannian gravity models $(Q \neq 0, T \neq 0$, $R \neq 0$ ) can be found in the literature [1]-[7]. An important subclass of these models is provided by Einstein-Cartan-Dirac theory written in a Riemann-Cartan space-time in which a spinor field is the source of space-time torsion [8] and the torsion may contribute to neutrino oscillations [9]. We emphasize that the connection is metric compatible $(Q=0)$ in such theories. In certain modifications of this model, curvature together with non-metricity is also constrained to zero, but torsion is left nonzero. These theories are called teleparallel gravity models (see [10]-14] and references therein).

Another approach to non-Riemannian gravity is due to $\mathrm{H}$. Weyl who used only the trace of the non-metricity 1-form, i.e. Weyl 1-form, and in an unsuccessful attempt interpreted that as the electromagnetic potential 1-form. In addition to this pioneering work, there have been other theoretical models in space-times with $Q \neq 0, R \neq 0, T=0$ ([15]-[16] and references therein). On the other hand, gravity models in the space-times with $Q \neq 0, T=0, R=0$, that we are going to call symmetric teleparallel gravity (STPG), have not been studied that much [17-[18. This paper aims to fill this gap. Due to the fact that curvature and torsion vanish, it is usually asserted that this model is a gravitational field theory that is closest possible to flat space-time. This is an interesting aspect of STPG, that deserves further analysis.

After recalling some mathematical preliminaries in Sec.2 we derive the field equations of STPG from the most general quadratic non-metricity Lagrangian in Sec 3. Mathematical details of the variations are given in an appendix. Then in Sec 3.1 we write down the nontrivial dynamical gravitational field equations for a spherically symmetric background metric and find a class of solutions that includes a Schwarzschild-type solution, in which we identify the integration constant with a spherically symmetric mass located at the coordinate center; Schwarzschild-de Sitter-type, in which we we identify one of the integration constants with the cosmological constant, and a Reissner-Nordström-type solution. The last section is reserved for the conclusion. We speculate that dark matter may couple to a new kind of gravitational charge and this speculative charge may interact gravitationally only through non-metricity. Here we adhere to the following conventions: Indices denoted by Greek letters $\alpha, \beta, \cdots=\hat{0}, \hat{1}, \hat{2}, \hat{3}$ are holonomic or coordinate indices, $a, b, \cdots=0,1,2,3$ are anholonomic or frame indices. We abbreviate the wedge products of orthonormal co-frames $e^{a b \cdots}=e^{a} \wedge e^{b} \wedge \cdots$ and denote symmetric and anti-symmetric indices as $(a b)=1 / 2(a+b)$ and $[a b]=1 / 2(a-b)$.

\section{Mathematical preliminaries}

Spacetime is denoted by the triple $\{M, g, \nabla\}$ where $\mathrm{M}$ is a 4-dimensional differentiable manifold equipped with a Lorentzian metric $g$ which is a second rank, covariant, symmetric, non-degenerate tensor and $\nabla$ is a linear connection which defines parallel transport of vectors (or more generally tensors and spinors). In terms of an orthonormal basis $\left\{X_{a}\right\}$, the metric can be written as

$$
g=\eta_{a b} e^{a} \otimes e^{b} \quad, \quad a, b, \cdots=0,1,2,3
$$


where $\eta_{a b}=(-,+,+,+)$ is the Minkowski metric and $\left\{e^{a}\right\}$ is the orthonormal co-frame. The local orthonormal frame $\left\{X_{a}\right\}$ is dual to the co-frame $\left\{e^{a}\right\}$;

$$
e^{b}\left(X_{a}\right)=\imath_{a} e^{b}=\delta_{a}^{b}
$$

where $\imath_{X_{a}} \equiv \imath_{a}$ are the interior product operators. The space-time orientation is set by the choice $\epsilon_{0123}=+1$ or $* 1=e^{0123}$ where $*$ denotes the Hodge dual map. In addition, the connection is specified by a set of connection 1-forms $\left\{\Lambda_{b}^{a}\right\}$. In the gauge approach to gravity, $\eta_{a b}, e^{a}, \quad \Lambda_{b}^{a}$ are interpreted as the generalized gauge potentials, while the corresponding field strengths; the nonmetricity 1-forms, torsion 2-forms and curvature 2-forms are defined through the Cartan structure equations.

\subsection{Nonmetricity : $\mathrm{Q}_{\mathrm{b}}^{\mathrm{a}}$}

$$
2 Q_{a b}:=-D \eta_{a b}=\Lambda_{a b}+\Lambda_{b a}
$$

where $D$ denotes the covariant exterior derivative. When $Q_{a b}=0$, it is said that the connection is metric compatible. In this case $\Lambda_{a b}=-\Lambda_{b a}$. Geometrically the non-metricity tensor measures the deformation of length and angle standards during parallel transport. Technically speaking it is a measure of compatibility of the affine connection with the metric. The scalar product of vectors is, in general, not preserved during parallel transport due to the appearance of non-metricity. As a result if we parallel transport a vector along a closed curve, the length of the final vector will be different from that of the initial vector.

\subsection{Torsion : $\mathrm{T}^{\mathrm{a}}$}

$$
T^{a}:=D e^{a}=d e^{a}+\Lambda_{b}^{a} \wedge e^{b}
$$

where $d$ denotes exterior derivative. If the connection is not only metric compatible, but the torsion is also zero, i.e, both $Q_{a b}=0$ and $T^{a}=0$, it is said that the connection is Levi-Civita. Geometrically it relates to the translational group. If a vector (say $A=X_{a} A^{a}$ ) is parallel transported around a loop by means of the linear connection $\Lambda_{b}^{a}$, a transformation of $A$ is induced, the linear piece of which is determined by the curvature and the translational piece by the torsion. To see its translational part let us consider parallel transport. Suppose that a vector $A=A^{\mu} \partial_{\mu}$ is parallelly transported according to the following prescription: First parallel transport the vector along $d x^{\mu}$ and then along $d x^{\nu}$. Compare that with repeating the trip in reverse order, that is, first transport the vector along $d x^{\nu}$ and then along $d x^{\mu}$. In flat space-time this series of actions sketches out a parallelogram. What happens in curved space? As we will see later, with zero torsion one still obtains a parallelogram, but of course the vector ends up rotated. With non-zero torsion something else happens; the two different routes do not bring the vector to the same point. The total difference between the initial and the final points of the vector, $\Delta A^{a}$, is given by

$$
\Delta A^{a} \simeq \frac{1}{2} \int_{S} T_{\mu \nu}^{a} d x^{\mu} \wedge d x^{\nu}
$$

where $S$ is the surface enclosed by the loop. It is sometimes said that closed parallelograms do not exist in space-time with torsion. 


\section{$2.3 \quad$ Curvature : $\mathrm{R}_{\mathrm{b}}^{\mathrm{a}}$}

$$
R_{b}^{a}:=D \Lambda_{b}^{a}:=d \Lambda_{b}^{a}+\Lambda_{c}^{a} \wedge \Lambda_{b}^{c}
$$

Geometrically it relates to the linear group. Now let us see the effect of curvature on vectors after being parallel transported along a closed loop. If the vector does not undergo a rotation, then space is flat. Conversely, if the vector is rotated, then space is curved. Performing the parallel transport of a vector $A=X_{a} A^{a}$ around a closed small path one obtains the following transformation

$$
\Delta A^{a} \simeq \frac{1}{2} \int_{S} R_{b \mu \nu}^{a} A^{b} d x^{\mu} \wedge d x^{\nu}
$$

where $S$ is the surface of the loop. Space-time is curved if this tensor is not zero, and the space-time is flat if this tensor is zero. Physically space-time curvature is a fundamental tensor in gravitation, in particular in Einstein's general relativity.

These field strengths satisfy the Bianchi identities

$$
\begin{aligned}
D Q_{a b} & =\frac{1}{2}\left(R_{a b}+R_{b a}\right), \\
D T^{a} & =R^{a}{ }_{b} \wedge e^{b}, \\
D R_{b}^{a} & =0 .
\end{aligned}
$$

We also need the identities ${ }^{1}$

$$
\begin{aligned}
D * e_{a} & =-Q \wedge * e_{a}+T^{b} \wedge * e_{a b} \\
D * e_{a b} & =-Q \wedge * e_{a b}+T^{c} \wedge * e_{a b c} \\
D * e_{a b c} & =-Q \wedge * e_{a b c}+T^{d} \wedge * e_{a b c d} \\
D * e_{a b c d} & =-Q \wedge * e_{a b c d}
\end{aligned}
$$

where $Q=\Lambda_{a}^{a}=Q^{a}{ }_{a}$ is the Weyl 1-form. The linear connection 1-forms can be decomposed uniquely as follows [2]-4]:

$$
\Lambda_{b}^{a}=\omega_{b}^{a}+K_{b}^{a}+q_{b}^{a}+Q_{b}^{a}
$$

where $\omega^{a}{ }_{b}$ are the Levi-Civita connection 1-forms

$$
d e^{a}+\omega_{b}^{a} \wedge e^{b}=0
$$

$K^{a}{ }_{b}$ are the contortion 1-forms

$$
K^{a}{ }_{b} \wedge e^{b}=T^{a},
$$

and $q^{a}{ }_{b}$ are the anti-symmetric tensor 1-forms

$$
q_{a b}=-\left(\imath_{a} Q_{b c}\right) \wedge e^{c}+\left(\iota_{b} Q_{a c}\right) \wedge e^{c} .
$$

In the above decomposition the symmetric part

$$
\Lambda_{(a b)}=Q_{a b}
$$

\footnotetext{
${ }^{1}$ Since $Q^{a b}=\frac{1}{2} D \eta^{a b} \neq 0$ we pay special attention in lowering and raising an index in front of the covariant exterior derivative.
} 
while the anti-symmetric part

$$
\Lambda_{[a b]}=\omega_{a b}+K_{a b}+q_{a b} .
$$

It is cumbersome to take into account all components of non-metricity in gravitational models. Therefore we will be content with dealing only with certain irreducible parts of it to gain physical insight. The irreducible decompositions of non-metricity invariant under the Lorentz group are summarily given below [4. The non-metricity 1-forms $Q_{a b}$ can be split into their trace-free $\bar{Q}_{a b}$ and the trace parts as

$$
Q_{a b}=\bar{Q}_{a b}+\frac{1}{4} \eta_{a b} Q
$$

where the Weyl 1-form $Q=Q^{a}{ }_{a}$ and $\eta^{a b} \bar{Q}_{a b}=0$. Let us define

$$
\begin{aligned}
\Lambda_{b} & :=\imath_{a} \bar{Q}_{b}^{a}, \quad \Lambda:=\Lambda_{a} e^{a}, \\
\Theta_{b} & :=*\left(\bar{Q}_{a b} \wedge e^{a}\right), \quad \Theta:=e^{b} \wedge \Theta_{b}, \quad \Omega_{a}:=\Theta_{a}-\frac{1}{3} \imath_{a} \Theta
\end{aligned}
$$

as to use them in the decomposition of $Q_{a b}$ as

$$
Q_{a b}={ }^{(1)} Q_{a b}+{ }^{(2)} Q_{a b}+{ }^{(3)} Q_{a b}+{ }^{(4)} Q_{a b}
$$

where

$$
\begin{aligned}
{ }^{(2)} Q_{a b} & =\frac{1}{3} *\left(e_{a} \wedge \Omega_{b}+e_{b} \wedge \Omega_{a}\right), \\
{ }^{(3)} Q_{a b} & =\frac{2}{9}\left(\Lambda_{a} e_{b}+\Lambda_{b} e_{a}-\frac{1}{2} \eta_{a b} \Lambda\right), \\
{ }^{(4)} Q_{a b} & =\frac{1}{4} \eta_{a b} Q \\
{ }^{(1)} Q_{a b} & =Q_{a b}-{ }^{(2)} Q_{a b}-{ }^{(3)} Q_{a b}-{ }^{(4)} Q_{a b} .
\end{aligned}
$$

We have

$$
\begin{aligned}
\eta_{a b}{ }^{(1)} Q^{a b}=\eta_{a b}{ }^{(2)} Q^{a b}=\eta_{a b}{ }^{(3)} Q^{a b} & =0, \\
\imath_{a}{ }^{(1)} Q^{a b}=\imath_{a}{ }^{(2)} Q^{a b} & =0, \\
e_{a} \wedge{ }^{(1)} Q^{a b} & =0, \\
\imath_{(a}{ }^{(2)} Q_{b c)} & =0 .
\end{aligned}
$$

Thus the components are orthogonal in the following sense

$$
{ }^{(i)} Q^{a b} \wedge *^{(j)} Q_{a b}=\delta^{i j} N_{i j} \quad \text { (no summation over } i j \text { ) }
$$

where $\delta^{i j}$ is the Kronecker symbol and $N_{i j}$ is a 4 -form. Then

$$
\begin{aligned}
& { }^{(1)} Q^{a b} \wedge *{ }^{(1)} Q_{a b}=Q^{a b} \wedge * Q_{a b}-{ }^{(2)} Q^{a b} \wedge *{ }^{(2)} Q_{a b}-{ }^{(3)} Q^{a b} \wedge *{ }^{(3)} Q_{a b} \\
& { }^{(4)} Q^{a b} \wedge *^{(4)} Q_{a b} \text {, } \\
& { }^{(2)} Q^{a b} \wedge *^{(2)} Q_{a b}=\frac{2}{3}\left(Q_{a c} \wedge e^{a}\right) \wedge *\left(Q^{b c} \wedge e_{b}\right)-\frac{2}{9}\left(\imath^{a} Q_{a c}\right)\left(\imath_{b} Q^{b c}\right) * 1-\frac{2}{9} Q \wedge * Q \\
& +\frac{4}{9}\left(\imath_{a} Q\right)\left(\imath_{b} Q^{a b}\right) * 1 \\
& { }^{(3)} Q^{a b} \wedge *^{(3)} Q_{a b}=\frac{4}{9}\left(\imath^{a} Q_{a c}\right)\left(\iota_{b} Q^{b c}\right) * 1+\frac{1}{36} Q \wedge * Q-\frac{2}{9}\left(\imath_{a} Q\right)\left(\imath_{b} Q^{a b}\right) * 1, \\
& { }^{(4)} Q^{a b} \wedge *^{(4)} Q_{a b}=\frac{1}{4} Q \wedge * Q .
\end{aligned}
$$




\section{$3 \quad$ Symmetric teleparallel gravity}

We formulate STPG in terms of a Lagrangian 4-form

$$
\mathcal{L}=L+\lambda_{a} \wedge T^{a}+R^{a}{ }_{b} \wedge \rho_{a}{ }^{b}
$$

where $\rho_{a}{ }^{b}$ and $\lambda_{a}$ are the Lagrange multiplier 2-forms giving the constraints

$$
R_{b}^{a}=0 \quad, \quad T^{a}=0 .
$$

$\mathcal{L}$ changes by a closed form under the transformations

$$
\begin{aligned}
\lambda_{a} & \rightarrow \lambda_{a}+D \mu_{a}, \\
\rho_{a}{ }^{b} & \rightarrow \rho_{a}{ }^{b}+D \xi_{a}{ }^{b}-\mu_{a} \wedge e^{b}
\end{aligned}
$$

of the Lagrange multiplier fields. Here $\mu_{a}$ and $\xi_{a}{ }^{b}$ are arbitrary 1-forms. To show this invariance we use the Bianchi identities (8)-(10) and discard exact forms. Consequently the field equations derived from the Lagrangian 4-form (34) will determine the Lagrange multipliers only up to above transformations. The gravitational field equations are derived from (34) by independent variations with respect to the connection $\left\{\Lambda_{b}^{a}\right\}$ and the ortohonormal co-frame $\left\{e^{a}\right\}$ 1-forms, respectively:

$$
\begin{array}{r}
\lambda_{a} \wedge e^{b}+D \rho_{a}{ }^{b}=-\Sigma_{a}{ }^{b}, \\
D \lambda_{a}=-\tau_{a}
\end{array}
$$

where $\Sigma_{a}{ }^{b}=\frac{\partial L}{\partial \Lambda^{a} b}$ and $\tau_{a}=\frac{\partial L}{\partial e^{a}}$. In principle the first field equation (38) is used to solve for the Lagrange multipliers $\lambda_{a}$ and $\rho_{a}{ }^{b}$ and the second field equation (39) governs the dynamics of the gravitational fields. Here the first equation, however, has 64 and the second one has 16 independent components, thus giving the total number of independent equations 80 . On the other hand, there are totally 120 unknowns: 24 for $\lambda_{a}$ plus 96 for $\rho_{a}{ }^{b}$. But we note that the left-hand side of (38) is invariant under the transformations (36)-(37) and consequently it is sufficient to determine the gauge invariant piece of the Lagrange multipliers, namely $\lambda_{a} \wedge e^{b}+D \rho_{a}{ }^{b}$, in terms of $\Sigma_{a}{ }^{b}$. It is important to notice $D \lambda_{a}$ rather than the Lagrange multipliers themselves couple to the second field equations (39). As a result we must calculate $D \lambda_{a}$ directly and we can manage that by taking the set of covariant exterior derivative of (38):

$$
D \lambda_{a} \wedge e^{b}=-D \Sigma_{a}^{b}
$$

Here we used the constraints

$$
\begin{aligned}
D e^{b} & =T^{b}=0 \\
D^{2} \rho_{a}{ }^{b} & =D\left(D \rho_{a}{ }^{b}\right)=R^{b}{ }_{c} \wedge \rho_{a}{ }^{c}-R^{c}{ }_{a} \wedge \rho_{c}{ }^{b}=0
\end{aligned}
$$

where the covariant exterior derivative of a $(1,1)$-type tensor is

$$
D \rho_{a}^{b}=d \rho_{a}^{b}+\Lambda_{c}^{b} \wedge \rho_{a}^{c}-\Lambda_{a}^{c} \wedge \rho_{c}^{b}
$$

The result (40) is unique because $D \lambda_{a} \rightarrow D \lambda_{a}$ under (36). One can consult Ref. [19] for further discussions on gauge symmetries of Lagrange multipliers. Thus we arrive at the field equation

$$
D \Sigma_{a}{ }^{b}-\tau_{a} \wedge e^{b}=0
$$


Now we write down the following Lagrangian 4-form which is the most general quadratic expression in the non-metricity tensor [20]:

$$
L=\frac{1}{2 \kappa}\left[k_{0} R^{a}{ }_{b} \wedge * e_{a}{ }^{b}+\sum_{I=1}^{4} k_{I}^{(I)} Q_{a b} \wedge *^{(I)} Q^{a b}+k_{5}\left({ }^{(3)} Q_{a b} \wedge e^{b}\right) \wedge *\left({ }^{(4)} Q^{a c} \wedge e_{c}\right)\right]
$$

Here $k_{0}, k_{1}, k_{2}, k_{3}, k_{4}, k_{5}$ are dimensionless coupling constants and $\kappa=\frac{8 \pi G}{c^{3}}$, with $G$ the Newton's gravitational constant. Inserting (30)-(33) into (45) we find

$$
\begin{array}{r}
L=\frac{1}{2 \kappa}\left[k_{0} R_{b}^{a} \wedge * e_{a}{ }^{b}+c_{1} Q_{a b} \wedge * Q^{a b}+c_{2}\left(Q_{a c} \wedge e^{a}\right) \wedge *\left(Q^{b c} \wedge e_{b}\right)\right. \\
\left.+c_{3}\left(\imath_{a} Q^{a c}\right)\left(\imath^{b} Q_{b c}\right) * 1+c_{4} Q \wedge * Q+c_{5}\left(\imath_{a} Q\right)\left(\imath_{b} Q^{a b}\right) * 1\right]
\end{array}
$$

where the new coefficients are the following combinations of the original coupling constants:

$$
\begin{aligned}
c_{1} & =k_{1}, \\
c_{2} & =-\frac{2}{3} k_{1}+\frac{2}{3} k_{2}, \\
c_{3} & =-\frac{2}{9} k_{1}-\frac{2}{9} k_{2}+\frac{4}{9} k_{3}, \\
c_{4} & =-\frac{1}{18} k_{1}-\frac{2}{9} k_{2}+\frac{1}{36} k_{3}+\frac{1}{4} k_{4}+\frac{1}{16} k_{5}, \\
c_{5} & =-\frac{2}{9} k_{1}+\frac{4}{9} k_{2}-\frac{2}{9} k_{3}-\frac{1}{4} k_{5} .
\end{aligned}
$$

When we use the results derived in the appendix by keeping in mind $Q^{a b}=\Lambda^{(a b)}$, together with (44) we obtain the variational field equations from (46)

$$
\Sigma_{a}{ }^{b}=\sum_{i=0}^{5} c_{i}{ }^{i} \Sigma_{a}{ }^{b} \quad, \quad \tau_{a}=\sum_{i=0}^{5} c_{i}{ }^{i} \tau_{a}
$$

where

$$
\begin{aligned}
{ }^{0} \Sigma_{a}{ }^{b} & =2 Q^{b c} \wedge * e_{a c}-Q \wedge * e_{a}{ }^{b}+T_{c} \wedge * e_{a}{ }^{b c} \\
{ }^{1} \Sigma_{a}{ }^{b} & =*\left(Q_{a}{ }^{b}+Q^{b}{ }_{a}\right) \\
{ }^{2} \Sigma_{a}{ }^{b} & =e_{a} \wedge *\left(Q^{b c} \wedge e_{c}\right)+e^{b} \wedge *\left(Q_{a c} \wedge e^{c}\right) \\
{ }^{3} \Sigma_{a}{ }^{b} & =\imath^{c} Q_{a c} * e^{b}+\imath_{c} Q^{b c} * e_{a} \\
{ }^{4} \Sigma_{a}{ }^{b} & =2 \delta_{a}^{b} * Q \\
{ }^{5} \Sigma_{a}{ }^{b} & =\frac{1}{2}\left(\imath^{b} Q\right) * e_{a}+\frac{1}{2}\left(\imath_{a} Q\right) * e^{b}+\delta_{a}^{b}\left(\imath_{c} Q^{c d}\right) * e_{d} \\
{ }^{0} \tau_{a} & =R^{b} \wedge * e_{a b}{ }^{c} \\
{ }^{1} \tau_{a} & =-\left(\imath_{a} Q^{b c}\right) \wedge * Q_{b c}-Q^{b c} \wedge\left(\imath_{a} * Q_{b c}\right) \\
{ }^{2} \tau_{a} & =-Q_{a b} \wedge *\left(Q^{b c} \wedge e_{c}\right)-\left(\imath_{a} Q^{c d}\right) e_{c} \wedge *\left(Q_{b d} \wedge e^{b}\right)+\left(Q_{b d} \wedge e^{b}\right) \wedge *\left(Q^{c d} \wedge e_{c a}\right) \\
{ }^{3} \tau_{a} & =-2\left(\imath_{a} Q^{b d}\right)\left(\imath^{c} Q_{c d}\right) * e_{b}+\left(\imath_{b} Q^{b d}\right)\left(\imath^{c} Q_{c d}\right) * e_{a} \\
{ }^{4} \tau_{a} & =-\left(\imath_{a} Q\right) * Q-Q \wedge\left(\imath_{a} * Q\right) \\
{ }^{5} \tau_{a} & =\left(\imath_{b} Q\right)\left(\imath_{c} Q^{b c}\right) * e_{a}-\left(\imath_{a} Q\right)\left(\imath_{c} Q^{b c}\right) * e_{b}-\left(\imath_{b} Q\right)\left(\imath_{a} Q^{b c}\right) * e_{c} .
\end{aligned}
$$

Since ${ }^{0} \tau_{a}=R^{b}{ }_{c} \wedge * e_{a b}{ }^{c}=0$ and $D^{0} \Sigma_{a}{ }^{b}=D^{2} * e_{a}{ }^{b} \sim R_{a}{ }^{b}=0$ we drop the Einstein-Hilbert term: $k_{0}=0$. The case $k_{0} \neq 0$ and others $=0$ is discussed in [18]. 


\subsection{Spherical symmetric solution to the model}

We now proceed the attempt for finding a solution to the STPG model. We naturally start dealing with the case of spherical symmetry for realistic simplicity. As usual in the study of exact solutions one has to choose the convenient local coordinates and make corresponding ansatz for the dynamical fields. We try to find a spherically symmetric solution with the line element

$$
g=-F^{2} d t^{2}+G^{2} d r^{2}+r^{2} d \theta^{2}+r^{2} \sin ^{2} \theta d \varphi^{2}
$$

where $F=F(r)$ and $G=G(r)$. A convenient choice for a tetrad reads

$$
e^{0}=F d t, \quad e^{1}=G d r, \quad e^{2}=r d \theta, \quad e^{3}=r \sin \theta d \varphi
$$

In addition to orthonormal co-frame choice, for the non-Riemannian connection we choose

$$
\begin{aligned}
& \Lambda_{12}=-\Lambda_{21}=-\frac{1}{r} e^{2}, \quad \Lambda_{13}=-\Lambda_{31}=-\frac{1}{r} e^{3}, \quad \Lambda_{23}=-\Lambda_{32}=-\frac{\cot \theta}{r} e^{3}, \\
& \Lambda_{00}=\frac{F^{\prime}}{F G} e^{1}, \quad \Lambda_{11}=\Lambda_{22}=\Lambda_{33}=\frac{1}{r}\left(1-\frac{1}{G}\right) e^{1}, \quad \text { others }=0 .
\end{aligned}
$$

Here prime denotes the derivative with respect to $r$. This gauge configuration satisfies the constraint equations $R_{b}^{a}(\Lambda)=0 \quad, \quad T^{a}(\Lambda)=0$. Equations(62)-(63) read explicitly

$$
\begin{aligned}
& \omega_{01}=-\frac{F^{\prime}}{F G} e^{0}, \quad \omega_{12}=-\frac{1}{r G} e^{2}, \quad \omega_{13}=-\frac{1}{r G} e^{3}, \quad \omega_{23}=-\frac{\cot \theta}{r} e^{3}, \\
& Q_{00}=\frac{F^{\prime}}{F G} e^{1}, \quad Q_{11}=Q_{22}=Q_{33}=\frac{1}{r}\left(1-\frac{1}{G}\right) e^{1}, \quad \text { others }=0 .
\end{aligned}
$$

Under this configuration the only nontrivial field equation comes from the trace of (44):

$$
d \Sigma_{a}^{a}+e^{a} \wedge \tau_{a}=0
$$

Symmetric and antisymmetric parts of the field equation give trivially zero. From (65) we obtain

$$
\begin{aligned}
-\frac{\ell_{1}}{G}\left(\frac{F^{\prime}}{F G}\right)^{\prime}-\frac{\ell_{2}}{G}\left(\frac{1-G}{r G}\right)^{\prime}+\ell_{3}\left(\frac{F^{\prime}}{F G}\right)^{2} & +\ell_{4}\left(\frac{F^{\prime}}{F G}\right)\left(\frac{1-G}{r G}\right)-\frac{2 \ell_{1}}{r G}\left(\frac{F^{\prime}}{F G}\right) \\
& -\frac{2 \ell_{2}}{r G}\left(\frac{1-G}{r G}\right)+\ell_{5}\left(\frac{1-G}{r G}\right)^{2}=0
\end{aligned}
$$

where

$$
\begin{aligned}
\ell_{1} & =2 c_{1}+2 c_{2}+8 c_{4}+c_{5}, \\
\ell_{2} & =6 c_{1}+4 c_{2}+2 c_{3}+24 c_{4}+7 c_{5}, \\
\ell_{3} & =-6 c_{4}-c_{5} \\
\ell_{4} & =-6 c_{1}-4 c_{2}-2 c_{3}-12 c_{4}-5 c_{5}, \\
\ell_{5} & =6 c_{1}+4 c_{2}+2 c_{3}+18 c_{4}+6 c_{5} .
\end{aligned}
$$

Due to the observational success of Schwarzschild solution of general relativity, we investigate solutions with $G=1 / F$. Then

$$
-\ell_{1} F F^{\prime \prime}+\ell_{3}\left(F^{\prime}\right)^{2}-\left(2 \ell_{1}+\ell_{2}-\ell_{4}\right) \frac{F F^{\prime}}{r}+\left(\ell_{5}-\ell_{2}\right) \frac{F^{2}}{r^{2}}-\ell_{4} \frac{F^{\prime}}{r}+\left(\ell_{2}-2 \ell_{5}\right) \frac{F}{r^{2}}+\ell_{5} \frac{1}{r^{2}}=0 .
$$


We can not find an analytical exact solution of this nonlinear second order differential equation. Therefore, we treat the linear sector of the equation

$$
r^{2}\left(F^{2}\right)^{\prime \prime}+\left(2+\frac{\ell_{2}}{\ell_{1}}\right) r\left(F^{2}\right)^{\prime}+\frac{\ell_{2}}{\ell_{1}} F^{2}=\frac{\ell_{2}}{\ell_{1}}
$$

by choosing our parameters as follows;

$$
\ell_{3}=-\ell_{1} \quad, \quad \ell_{4}=0 \quad, \quad \ell_{5}=\frac{\ell_{2}}{2}
$$

Here some special cases deserve attention.

1. For $\ell_{2}=\ell_{1}$, we obtain the solution

$$
F^{2}=1+\frac{C_{1}}{r}+D_{1} \frac{\ln r}{r}
$$

which is asymptotically flat; $\lim _{r \rightarrow \infty} F=1$. Here $C_{1}$ and $D_{1}$ are integration constants.

2. For $\ell_{2} \neq \ell_{1}$, the solution is found as

$$
F^{2}=1+\frac{C_{2}}{r}+\frac{D_{2}}{r^{\ell_{2} / \ell_{1}}}
$$

where $C_{2}$ and $D_{2}$ are integration constants.

(a) For $\ell_{2}=0$, we obtain a Schwarzschild-type solution with $D_{2}=0$ for asymptotically flatness and we identify the other constant with a spherically symmetric mass centered at the origin; $C_{2}=-2 M$.

(b) For $\ell_{2}=-2 \ell_{1}$, we obtain a Schwarzschild-de Sitter-type solution. We again identify $C_{2}$ with mass $C_{2}=-2 M$ and $D_{2}$ with cosmological constant ${ }^{2} D_{2}=-\frac{1}{3} \Lambda$. The $\Lambda$ term corresponds to a repulsive central force of magnitude $\frac{1}{3} \Lambda r$, which is independent of the central mass.

(c) For $\ell_{2}=2 \ell_{1}$, we obtain a Reissner-Nordström-type solution. We again identify $C_{2}$ with mass $C_{2}=-2 M$ while $D_{2}$ with a new kind of gravitational charge. We hope that besides ordinary matter that interacts gravitationally through its mass, the dark matter in the Universe may interact gravitationally through both its mass and this new gravitational charge.

\section{Conclusion}

Einstein's GR provides a (pseudo-)Riemannian formulation of gravitation in the absence of matter. The field equations are obtained as the local extremum of the Einstein-Hilbert action integral under metric variations. The integrand of this action is simply the curvature scalar of the spacetime curvature written in terms of the Levi-Civita connection. Such a connection is torsion-free and metric compatible and provides a useful reference connection because it depends entirely on the metric. Einstein's GR is very successful for describing the large scale structure of gravitational phenomena. But in spite of that, supergravity and superstring theories have suggested a more general geometry with torsion and non-metricity. At the level of effective theories there are hints that

\footnotetext{
${ }^{2}$ Current estimates suggest $|\Lambda|<10^{-54} \mathrm{~cm}^{-2}$, and this makes $\Lambda$ quite negligible in all noncosmological situations.
} 
a non-Riemannian geometry may offer a more economical and more elegant description of gravitational interactions. Although theories in which non-Riemannian geometrical fields are dynamical in the absence of matter are more difficult to interpret, they may play an important role in certain astrophysical contexts. In this context here we have studied the Lagrange formulation of the general symmetric teleparallel gravity model, a theory in which only non-metricity is nonvanishing. We have not seen a similar analysis of STPG in the literature. We consider the full 5-parameter symmetric teleparallel Lagrangian without a priori restricting the coupling constants $c_{1}, c_{2}, c_{3}, c_{4}, c_{5}$. The main motivation for this was to determine the place and significance of STPG in the framework of non-Riemannian models. We found that the field equations accept a Schwarzschild-type solution for some special values of the coupling constants. For some other set of values of the parameters we found a Schwarzscild-de Sitter-type solution. A further different set of values of the coupling constants yields a Reissner-Nordström-type solution. Consequently, we suggest that in addition to ordinary matter that interacts gravitationally through its mass, the dark matter in the Universe may interact gravitationally through both its mass and a new kind of gravitational charge [21]-[22]. The latter coupling is analogous to the coupling of electric charge to the photon where the analogue of the Maxwell field here is the non-metricity field strength. Such novel gravitational interactions may have a significant influence on the structure of black holes. For example, we may speculate that this unknown gravitational charge may have repulsive nature and thus black holes may not need to be close in one end. Possible matter couplings to the model and some physical events such as Berry phase and neutrino oscillations in STPG will be discussed elsewhere.

\section{Acknowledgment}

We are grateful to Prof. Dr. Tekin DERELI for fruitful discussions.

\section{A Calculus of variation}

Let $M$ be a $n$-dimensional manifold and $\alpha, \beta \in \Lambda^{p}(M)$ where $\Lambda^{p}(M)$ denotes any $p$-form on $M$. Then we would like to find the extremum of the following action integral

$$
I\left[\alpha, \beta, e^{a}\right]=\int_{M} \alpha \wedge * \beta
$$

by varying it with respect to the variables; $\alpha, \beta$ and $e^{a}$ :

$$
\delta I=\int_{M} \delta \alpha \wedge * \beta+\alpha \wedge \delta * \beta
$$

The second term on the right hand side needs a detailed calculation, because it contains the Hodge star.

$$
\begin{aligned}
\alpha \wedge \delta * \beta & =\alpha \wedge \delta\left(\frac{1}{p !} \beta_{i_{1} \cdots i_{p}} * e^{i_{1} \cdots i_{p}}\right) \\
& =\alpha \wedge \frac{1}{p !}\left(\delta \beta_{i_{1} \cdots i_{p}}\right) * e^{i_{1} \cdots i_{p}}+\alpha \wedge \frac{1}{p !} \beta_{i_{1} \cdots i_{p}} \delta * e^{i_{1} \cdots i_{p}}
\end{aligned}
$$

First use the identity in the first term $\theta \wedge * \gamma=\gamma \wedge * \theta$ where $\theta, \gamma \in \Lambda^{p}(M)$

$$
\alpha \wedge \delta * \beta=\frac{1}{p !}\left(\delta \beta_{i_{1} \cdots i_{p}}\right) e^{i_{1} \cdots i_{p}} \wedge * \alpha+\alpha \wedge \frac{1}{p !} \beta_{i_{1} \cdots i_{p}} \delta * e^{i_{1} \cdots i_{p}}
$$


and using

$$
\begin{aligned}
\delta \beta & =\delta\left(\frac{1}{p !} \beta_{i_{1} \cdots i_{p}} e^{i_{1} \cdots i_{p}}\right) \\
& =\frac{1}{p !}\left(\delta \beta_{i_{1} \cdots i_{p}}\right) e^{i_{1} \cdots i_{p}}+\left(\delta e^{i_{1}}\right) \wedge \frac{1}{(p-1) !} \beta_{i_{1} \cdots i_{p}} e^{i_{2} \cdots i_{p}} \\
& =\frac{1}{p !}\left(\delta \beta_{i_{1} \cdots i_{p}}\right) e^{i_{1} \cdots i_{p}}+\left(\delta e^{i_{1}}\right) \wedge\left(\imath_{i_{1}} \beta\right) \\
\frac{1}{p !}\left(\delta \beta_{i_{1} \cdots i_{p}}\right) e^{i_{1} \cdots i_{p}} & =\delta \beta-\left(\delta e^{a}\right) \wedge\left(\imath_{a} \beta\right)
\end{aligned}
$$

and the equality

$$
\begin{aligned}
& \frac{1}{p !} \beta_{i_{1} \cdots i_{p}} \delta * e^{i_{1} \cdots i_{p}}=\frac{1}{p !} \beta_{i_{1} \cdots i_{p}} \delta\left[\frac{1}{(n-p) !} \epsilon^{i_{1} \cdots i_{p}}{ }_{i_{p+1} \cdots i_{n}} e^{i_{p+1} \cdots i_{n}}\right] \\
& =\left(\delta e^{i_{p+1}}\right) \wedge \frac{1}{p !(n-p-1) !} \epsilon_{i_{p+1} \cdots i_{n}} \beta_{i_{1} \cdots i_{p}} e^{i_{p+2} \cdots i_{n}} \\
& =\left(\delta e^{a}\right) \wedge\left(\imath_{a} * \beta\right)
\end{aligned}
$$

in (80) and then substituting the result into (78) we obtain

$$
\begin{aligned}
\delta I & =\delta \int_{M} \alpha \wedge * \beta \\
& =\int_{M} \delta \alpha \wedge * \beta+\delta \beta \wedge * \alpha-\delta e^{a} \wedge\left[\left(\imath_{a} \beta\right) \wedge * \alpha-(-1)^{p} \alpha \wedge\left(\imath_{a} * \beta\right)\right]
\end{aligned}
$$

where $\alpha, \beta \in \Lambda^{p}(M)$.

$$
\text { Special Case: } 1 \quad \alpha=\beta=F=d A
$$

We encounter these kinds of Lagrangians in electromagnetic theory and teleparallel gravity models. In this case (84) becomes

$$
\delta I=\int_{M}(\delta d A) \wedge(2 * d A)-\delta e^{a} \wedge\left[\left(\imath_{a} F\right) \wedge * F-(-1)^{p} F \wedge\left(\imath_{a} * F\right)\right]
$$

where $A \in \Lambda^{p-1}(M)$. Since variation and exterior derivative commute with each other $\delta d=d \delta$, (85) may be rewritten as

$$
\begin{gathered}
\delta I=\int_{M}(d \delta A) \wedge(2 * d A)-\delta e^{a} \wedge\left[\left(\imath_{a} F\right) \wedge * F-(-1)^{p} F \wedge\left(\imath_{a} * F\right)\right] \\
=\int_{M}(\delta A) \wedge(-1)^{p}(2 d * F)+d(\delta A \wedge 2 * F) \\
\quad-\delta e^{a} \wedge\left[\left(\imath_{a} F\right) \wedge * F-(-1)^{p} F \wedge\left(\imath_{a} * F\right)\right]
\end{gathered}
$$

By applying the Stoke's theorem, the second term on the right hand side can be written as

$$
\int_{M} d(\delta A \wedge 2 * F)=\int_{\partial M} \delta A \wedge 2 * F=0
$$

because the boundary condition is $\left.\delta A\right|_{\partial M}=0$ where $\partial M$ is the boundary of $M$. Thus

$$
\begin{aligned}
\delta I & =\delta \int_{M} d A \wedge * d A \\
& =\int_{M}(\delta A) \wedge(-1)^{p}(2 d * F)-\delta e^{a} \wedge\left[\left(\imath_{a} F\right) \wedge * F-(-1)^{p} F \wedge\left(\imath_{a} * F\right)\right]
\end{aligned}
$$

where $F=d A \in \Lambda^{p}(M)$. 


Special Case:2 $\quad \alpha=e_{a} \wedge M^{a}$ and $\beta=e_{a} \wedge N^{a}$

These kinds of Lagrangians are encountered in non-Riemannian gravity models in which irreducible components of gauge fields are used. In this case (84) becomes

$$
\begin{aligned}
\delta I=\int_{M} \delta\left(e_{a} \wedge M^{a}\right) \wedge *\left(e_{b} \wedge N^{b}\right)+\delta\left(e_{a} \wedge N^{a}\right) \wedge *\left(e_{b} \wedge M^{b}\right) \\
-\delta e^{a} \wedge\left[\imath_{a}\left(e_{b} \wedge N^{b}\right) \wedge *\left(e_{c} \wedge M^{c}\right)-(-1)^{p}\left(e_{c} \wedge M^{c}\right) \wedge \imath_{a} *\left(e_{b} \wedge N^{b}\right)\right]
\end{aligned}
$$

where $M^{a}, N^{a} \in \Lambda^{p-1}(M)$. Organizing terms

$$
\begin{aligned}
\delta I= & \int_{M} \delta e_{a} \wedge M^{a} \wedge *\left(e_{b} \wedge N^{b}\right)+e_{a} \wedge \delta M^{a} \wedge *\left(e_{b} \wedge N^{b}\right) \\
& +\delta e_{a} \wedge N^{a} \wedge *\left(e_{b} \wedge M^{b}\right)+e_{a} \wedge \delta N^{a} \wedge *\left(e_{b} \wedge M^{b}\right) \\
& -\delta e^{a} \wedge\left[N_{a} \wedge *\left(e_{c} \wedge M^{c}\right)-e_{b} \wedge i_{a} N^{b} \wedge *\left(e_{c} \wedge M^{c}\right)\right. \\
& \left.-(-1)^{p}\left(e_{c} \wedge M^{c}\right) \wedge *\left(e_{b} \wedge N^{b} \wedge e_{a}\right)\right] .
\end{aligned}
$$

we obtain

$$
\begin{gathered}
\delta I=\delta \int_{M}\left(e_{a} \wedge M^{a}\right) \wedge *\left(e_{b} \wedge N^{b}\right) \\
=\int_{M} \delta M^{a} \wedge e_{a} \wedge *\left(N^{b} \wedge e_{b}\right)+\delta N^{a} \wedge e_{a} \wedge *\left(M^{b} \wedge e_{b}\right) \\
+\delta e^{a} \wedge\left[M_{a} \wedge *\left(e_{b} \wedge N^{b}\right)+e_{b} \wedge\left(e_{a} N^{b}\right) \wedge *\left(e_{c} \wedge M^{c}\right)\right. \\
\left.-\left(e_{c} \wedge M^{c}\right) \wedge *\left(e_{a} \wedge e_{b} \wedge N^{b}\right)\right] .
\end{gathered}
$$

Special Case: $3 \quad \alpha=\imath_{a} B^{a}$ and $\beta=\imath_{a} C^{a}$

In this case (84) becomes

$$
\begin{aligned}
\delta I=\int_{M} \delta\left(\imath_{a} B^{a}\right) & \wedge * \imath_{b} C^{b}+\delta\left(\imath_{a} C^{a}\right) \wedge * \iota_{b} B^{b} \\
-\delta e^{a} & \wedge\left[\left(\imath_{a} \iota_{b} C^{b}\right) \wedge *\left(\imath_{c} B^{c}\right)-(-1)^{p}\left(\imath_{c} B^{c}\right) \wedge\left(\imath_{a} * \iota_{b} C^{b}\right)\right]
\end{aligned}
$$

where $B^{a}, C^{a} \in \Lambda^{p+1}(M)$. First we write the first term on the right hand side as

$$
\delta\left(\imath_{a} B^{a}\right) \wedge * \iota_{b} C^{b}=\imath_{\delta a} B^{a} \wedge *\left(\iota_{b} C^{b}\right)+\imath_{a} \delta B^{a} \wedge *\left(\iota_{b} C^{b}\right),
$$

so that

$$
\begin{aligned}
\imath_{\delta a} B^{a} \wedge *\left(\imath_{b} C^{b}\right) & =\imath_{\delta a}\left(\frac{1}{(p+1) !} B^{a,{ }_{i_{1} \cdots i_{p+1}}} e^{i_{1} \cdots i_{p+1}}\right) \wedge *\left(\iota_{b} C^{b}\right) \\
& =\frac{1}{(p+1) !} B^{a}{ }_{{ }_{1} \cdots i_{p+1}}\left(\imath_{\delta a} e^{i_{1} \cdots i_{p+1}}\right) \wedge *\left(\iota_{b} C^{b}\right) \\
& =\left(\imath_{\delta a} e^{i_{1}}\right) \wedge \frac{1}{p !} B^{a,}{ }_{{ }_{1} \cdots i_{p+1}} e^{i_{2} \cdots i_{p+1}} \wedge *\left(\iota_{b} C^{b}\right) .
\end{aligned}
$$

By making the use of the duality

$$
\imath_{a} e^{b}=\delta_{a}^{b} \quad \rightarrow \quad \delta\left(\imath_{a} e^{b}\right)=0 \quad \rightarrow \quad \imath_{\delta a} e^{b}=-\imath_{a} \delta e^{b}
$$


this equation takes the form

$$
\imath_{\delta a} B^{a} \wedge *\left(\iota_{b} C^{b}\right)=-\left(\imath_{a} \delta e^{i_{1}}\right) \wedge \frac{1}{p !} B^{a,{ }_{i_{1} \cdots i_{p+1}}} e^{i_{2} \cdots i_{p+1}} \wedge *\left(\iota_{b} C^{b}\right) .
$$

Any $(n+1)$-form is equal to zero on a $n$-dimensional manifold, i.e.,

$$
\imath_{a}\left(-\delta e^{i_{1}} \wedge \frac{1}{p !} B^{a,{ }_{i_{1} \cdots i_{p+1}}} e^{i_{2} \cdots i_{p+1}} \wedge *\left(\iota_{b} C^{b}\right)\right)=0 .
$$

This implpies

$$
\begin{aligned}
& \imath_{\delta a} B^{a} \wedge *\left(\imath_{b} C^{b}\right)=(-1)^{p+1} \delta e^{i_{1}} \wedge \frac{1}{p !} B^{a,}{ }_{i_{1} \cdots i_{p+1}} e^{i_{2} \cdots i_{p+1}} \wedge \imath_{a} *\left(\iota_{b} C^{b}\right) \\
& -\delta e^{i_{1}} \wedge \frac{1}{(p-1) !} B^{a,}{ }_{i_{1} a i_{3} \cdots i_{p+1}} e^{i_{3} \cdots i_{p+1}} \wedge *\left(\iota_{b} C^{b}\right) \\
& =(-1)^{p+1} \delta e^{a} \wedge\left(\imath_{a} B^{c}\right) \wedge \imath_{c} *\left(\imath_{b} C^{b}\right)-\delta e^{a} \wedge\left(\imath_{c} \imath_{a} B^{c}\right) \wedge *\left(\imath_{b} C^{b}\right) \\
& =\delta e^{a} \wedge\left[\left(\imath_{a} \imath_{c} B^{c}\right) \wedge *\left(\imath_{b} C^{b}\right)-\left(\imath_{a} B^{c}\right) \wedge *\left(e_{c} \wedge \imath_{b} C^{b}\right)\right]
\end{aligned}
$$

where we used the identity $\imath_{a} * \Phi=*\left(\Phi \wedge e^{a}\right)$ for $\Phi \in \Lambda^{p}(M)$. Now we calculate the second term on the right hand side of (93) by forming a $(n+1)$-form on $n$-dimensional manifold

$$
\begin{aligned}
\imath_{a}\left[\delta B^{a} \wedge *\left(\imath_{b} C^{b}\right)\right] & =0 \\
\imath_{a} \delta B^{a} \wedge *\left(\imath_{b} C^{b}\right) & =\delta B^{a} \wedge *\left(e_{a} \wedge \imath_{b} C^{b}\right) .
\end{aligned}
$$

Then collecting (98) and (99), we write down (93)

$$
\delta\left(\imath_{a} B^{a}\right) \wedge * \imath_{b} C^{b}=\delta B^{a} \wedge *\left(e_{a} \wedge \imath_{b} C^{b}\right)+\delta e^{a} \wedge\left[\left(\imath_{a} \imath_{c} B^{c}\right) \wedge *\left(\iota_{b} C^{b}\right)-\left(\imath_{a} B^{c}\right) \wedge *\left(e_{c} \wedge \imath_{b} C^{b}\right)\right] .
$$

In order to calculate the second term on the right hand side of (92) we just replace $B$ with $C$ in equation (100):

$$
\delta\left(\imath_{a} C^{a}\right) \wedge * \imath_{b} B^{b}=\delta C^{a} \wedge *\left(e_{a} \wedge \imath_{b} B^{b}\right)+\delta e^{a} \wedge\left[\left(\imath_{a} \imath_{c} C^{c}\right) \wedge *\left(\imath_{b} B^{b}\right)-\left(\imath_{a} C^{c}\right) \wedge *\left(e_{c} \wedge \imath_{b} B^{b}\right)\right] .
$$

Thus, when we put all the results into (92), we find

$$
\begin{aligned}
\delta I=\delta \int_{M}\left(\imath_{a} B^{a}\right) \wedge *\left(\imath_{b} C^{b}\right) & \\
=\int_{M} \delta B^{a} \wedge *\left(e_{a} \wedge \imath_{b} C^{b}\right) & +\delta C^{a} \wedge *\left(e_{a} \wedge \imath_{b} B^{b}\right) \\
+\delta e^{a} \wedge\left[\left(\imath_{a} \imath_{c} B^{c}\right) \wedge\right. & *\left(\imath_{b} C^{b}\right)-\left(\imath_{a} B^{c}\right) \wedge *\left(e_{c} \wedge \imath_{b} C^{b}\right)-\left(\imath_{a} C^{c}\right) \wedge *\left(e_{c} \wedge \imath_{b} B^{b}\right) \\
& \left.+\left(\imath_{c} B^{c}\right) \wedge *\left(e_{a} \wedge \imath_{b} C^{b}\right)\right]
\end{aligned}
$$

where $B^{a}, C^{a} \in \Lambda^{p+1}(M)$.

$$
\text { Special Case: } 4 \quad \alpha=\imath_{a} Q^{a b} \text { and } \beta=\imath_{b} R
$$

In this case (84) becomes

$$
\begin{aligned}
\delta I=\int_{M} \delta\left(\imath_{a} Q^{a b}\right) & \wedge * \imath_{b} R+\delta\left(\iota_{b} R\right) \wedge * \iota_{b} Q^{a b} \\
-\delta e^{a} & \wedge\left[\left(\imath_{a} \iota_{b} R\right) \wedge *\left(\imath_{c} B^{b c}\right)-(-1)^{p}\left(\imath_{c} Q^{b c}\right) \wedge\left(\imath_{a} * \iota_{b} R\right)\right]
\end{aligned}
$$


where $Q^{a b}, R \in \Lambda^{p+1}(M)$. First we handle the first and the second terms on the right hand side

$$
\begin{aligned}
& \delta\left(\imath_{a} Q^{a b}\right) \wedge * \imath_{b} R=\imath_{\delta a} Q^{a b} \wedge *\left(\iota_{b} R\right)+\imath_{a} \delta Q^{a b} \wedge *\left(\iota_{b} R\right) \\
& =\delta Q^{a b} \wedge *\left(e_{a} \wedge \imath_{b} R\right)+\delta e^{a} \wedge\left[\left(\imath_{a} \imath_{b} Q^{b c}\right) \wedge *\left(\imath_{c} R\right)\right. \\
& \left.-\left(\imath_{a} Q^{b c}\right) \wedge *\left(e_{b} \wedge \imath_{c} R\right)\right], \\
& \delta\left(\imath_{b} R\right) \wedge *\left(\imath_{a} Q^{a b}\right)=\imath_{\delta a} R \wedge *\left(\iota_{b} Q^{a b}\right)+\imath_{b} \delta R \wedge *\left(\imath_{a} Q^{a b}\right) \\
& =\delta R \wedge *\left(e_{a} \wedge \imath_{b} Q^{a b}\right)+\delta e^{a} \wedge\left[\left(\imath_{a} \imath_{b} R\right) \wedge *\left(\imath_{c} Q^{b c}\right)\right. \\
& \left.-\left(\imath_{a} R\right) \wedge *\left(e_{b} \wedge \imath_{c} Q^{b c}\right)\right] \text {. }
\end{aligned}
$$

Thus

$$
\begin{aligned}
& \delta I=\delta \int_{M}\left(\imath_{a} Q^{a b}\right) \wedge *\left(\imath_{b} R\right) \\
& =\int_{M} \delta Q^{a b} \wedge *\left(e_{a} \wedge \imath_{b} R\right)+\delta R \wedge *\left(e_{a} \wedge \imath_{b} Q^{a b}\right) \\
& +\delta e^{a} \wedge\left[\left(\imath_{a} \imath_{b} Q^{b c}\right) \wedge *\left(\imath_{c} R\right)-\left(\imath_{a} Q^{b c}\right) \wedge *\left(e_{b} \wedge \imath_{c} R\right)-\left(\imath_{a} R\right) \wedge *\left(e_{b} \wedge \imath_{c} Q^{b c}\right)\right. \\
& \left.+\left(\imath_{c} Q^{b c}\right) \wedge *\left(e_{a} \wedge \imath_{b} R\right)\right]
\end{aligned}
$$

where $Q^{a b}, R \in \Lambda^{p+1}(M)$.

\section{References}

[1] M Adak, T Dereli and L H Ryder, Int. J. Mod. Phys. D12 (2003) 145-156 (arXiv:gr-qc/0208042)

[2] T Dereli and R W Tucker, Class. Quant. Grav. 4 (1987) 791

[3] T Dereli and R W Tucker, Class. Quant. Grav. 12 (1995) L31

[4] F W Hehl, J D McCrea, E W Mielke and Y Ne'eman, Phys. Rep. 258 (1995) 1

[5] R W Tucker and C Wang, Class. Quant. Grav. 12 (1995) 2587-2605

[6] T Dereli, M Önder, J Schray, R W Tucker and C Wang, Class. Quant. Grav. 13 (1996) L103L110 (arXiv:gr-qc/9604039)

[7] M Adak, T Dereli and L H Ryder Phys. Rev. D69 (2004) 123002 (arXiv:gr-qc/0303080)

[8] T Dereli and R W Tucker, J. Phys. A15 (1982) 1625

[9] M Adak, T Dereli and L H Ryder Class. Quant. Grav. 18 (2001) 1503-1512 (arXiv:gr-qc/0103046)

[10] K Hayashi and T Nakano, Prog. Theor. Phys. 38 (1967) 491

[11] K Hayashi and T Shirafuji, Phys. Rev. D19 (1979) 3524

[12] Y N Obukhov and J G Pereira, Phys. Rev. D67 (2003) 044016 (arXiv:gr-qc/0212080) 
[13] J W Maluf, Phys. Rev. D67 (2003) 108501 (arXiv:gr-qc/0304005)

[14] H I Arcos, V C De Andrade and J G Pereira, Int. J. Mod. Phys. D13 (2004) 807 (arXiv:gr-qc/0403074)

[15] T Dereli and R W Tucker, Class. Quant. Grav. 11 (1994) 2575-2583

[16] C Heinicke, P Baekler and F W Hehl, arXiv:gr-qc/0504005)

[17] J M Nester and H J Yo, Chinese J. Phys. 37 (1999) 113 (arXiv:gr-qc/9809049)

[18] M Adak and Ö Sert, Turk. J. Phys. 29 (2005) 1-7 (arXiv:gr-qc/0412007)

[19] M Blagojević and M Vasilić, Class. Quant. Grav. 17 (2000) 3785 (arXiv:hep-th/0006080)

[20] Y N Obukhov, E J Vlachynsky, W Esser and F W Hehl, Phys. Rev. D56 (1997) 7769

[21] R W Tucker and C Wang, Class. Quant. Grav. 15 (1998) 933-954

[22] T Dereli, M Önder, J Schray, R W Tucker and C Wang Non-Riemannian Gravitational Interactions: An Overview In the Proceedings of $9^{\text {th }}$ Marcel Grossmann Meeting, July 2000, Rome, Italy 